\title{
Optimal Maintenance Policy for a Technical System Subject to Hidden Faults and Randomly Occurring Hazards
}

\author{
Jacek Malinowski \\ Stochastic Methods Department, \\ Systems Research Institute, Polish Academy of Sciences, Warszawa, Poland. \\ E-mail: jacek.malinowski@ibspan.waw.pl
}

(Received June 12, 2020; Accepted September 8, 2020)

\begin{abstract}
The paper presents a method of finding the optimal time between inspections for a system subject to degradation-related faults which make the system vulnerable to randomly occurring external hazards that may cause its damage. Since faults are assumed to be hidden, periodic inspections and repairs have to be performed in order to detect and remove them. Otherwise, leaving the faulty system unmaintained would eventually lead to a very costly damage. It is also assumed that the time to occurrence of a fault is exponentially distributed and hazardous events constitute a Poisson process. The fault rate, the intensity of the Poisson process and the probability with which a hazardous event results in the system damage are the known parameters. The author presents two main results achieved by analyzing this maintenance model. First, the criteria to be fulfilled by the system parameters in order that preventive maintenance be cost-effective are given in the form of simple inequalities. These criteria must be met so that operating the system with preventive maintenance in place be less costly than operating it until a damage occurs and replacing it thereafter. Second, fairly simple equations are obtained from which the optimal time between inspections can be found numerically by the Newton-Raphson method. The analytical derivation of both the criteria and the equations is presented in detail and is the author's original work. To the best of his knowledge the obtained results are new in the area of maintenance modeling and analysis. For better understanding, theoretical considerations are illustrated by an example of a generic explosion prevention system.
\end{abstract}

Keywords- System fault, System damage, Damage-triggering factor, Long-run operating cost, Periodic maintenance, Optimal time between inspections.

\section{Introduction with the Literature Review}

This paper analyses a maintenance model of a technical system subject to degradation-related nonself-revealing faults and random occurrences of a hazardous event that, if the system is faulty, may trigger a catastrophic incident resulting in system damage. For example, an occasional spark from the electric installation in the vicinity of an LPG tank (a randomly occurring hazardous event) may set off an explosion (a catastrophic incident) if there is a leak in the tank (a hidden system fault). In order to reduce the chance of a catastrophe, the system should undergo periodic inspections and, in cases of fault detection, repairs or renewals should be carried out. A natural problem that arises here is how often the inspections should take place so that the system operating cost per unit time be minimal. Clearly, in order to cope with this problem, we need certain data about the system, i.e. the average hazardous-event-frequency, the distribution function of the system's TTF (time-tofault), the costs of inspection and preventive maintenance, the probability that the hazardous event causes damage to the faulty system, and the cost of such a damage. For the sake of analytical tractability it is assumed that TTF is exponentially distributed with the parameter $\mu$ and damagetriggering events constitute a homogenous Poisson process with the intensity $\lambda$.

The system's operation proceeds as follows. An inspection is planned to take place after time T from the last inspection or from the start of operation of a new or replaced system. If a fault is detected during an inspection, the system undergoes repair and is put back into operation. However, 
International Journal of Mathematical, Engineering and Management Sciences

Vol. 6, No. 1, 396-415, 2021

https://doi.org/10.33889/IJMEMS.2021.6.1.025

it can happen that an occurrence of a fault is followed by a hazardous event that precedes the next inspection and causes the system damage. Such a situation results in a very high damage cost, significantly higher than that of an inspection or repair. Each system damage is self-revealing, unlike a system fault, and is followed by replacement of the damaged system. The total operating cost involves inspection, repair, damage, and replacement costs. The research objective is to find the optimum $\mathrm{T}$ which minimizes the long run total operating cost per unit time. It turns out that such a $\mathrm{T}$ exists provided that the system parameters fulfill certain criteria formulated as simple inequalities, and it can be found from analytically obtained equations. The derivations of both the criteria and the equations for $\mathrm{T}$ are the author's main results constituting the main body of the paper.

The problem considered in this paper can be classified as that of optimizing an inspection and maintenance policy for a repairable technical system - one of the main issues in reliability engineering, which has long been a subject of extensive research. A comprehensive overview of this topic, along with a wide literature survey can be found in the following books: Gertsbakh (2000), Zequiera and Berenguer (2005), Jardine and Tsang (2006), and Duffuaa and Raouf (2015). Also, Vasili et al. (2011), Wang (2012, 2013), and Zhao et al. (2017) give a broad insight into many aspects of inspection and maintenance scheduling for various types of systems and policies. For some recent results in this area see Mendes et al. (2017), Badía et al. (2018), Sun et al. (2018), and Peng et al. (2019).

There exist numerous inspection/maintenance policies depending on the adopted models of the considered systems. They can be divided into two main categories: policies applied to single-unit systems, and those applied to multi-unit systems with particular reliability structure. Systems in the first category are often assumed to have a set (discrete or continuous) of degradation levels and the undertaken maintenance action is degradation-dependent. See Abdel-Hameed (1987, 1995), Nakagawa et al. (2010), Le and Tan (2013), Wang (2013), Guo et al. (2015), and Alaswad and Xiang (2017) for different models of one-unit systems and reviews of the respective literature. Comprehensive surveys of policies for multi-unit systems can be found in Nicolai and Dekker (2008), and Cao et al. (2018). As regards types of maintenance, Azadeh and Zadeh (2015) lay out the taxonomy of various maintenance policies, according to which the policy investigated in the current paper can be classified as age-based preventive maintenance.

The maintenance model proposed in this paper comprises the features of both a safety system and a delay-time model. In general terms, a safety system is a subsystem of a larger system whose task is to safeguard the latter against malfunction or damage. In our model, system fault can be regarded as the safety system failure which, in the case of a hazardous event, can be detrimental to the main system. The issue of safety systems maintenance is thoroughly outlined in Pascual et al. (2011), along with the analysis of a versatile model of such a system developed by the authors. In turn, the delay-time maintenance model is a concept introduced in Christer and Waller (1984). Delay-time is a (random) time between the first symptoms of the system degradation and the system failure. If an inspection performed during the delay-time reveals a degraded state, then the appropriate maintenance action is taken in order to avoid the impending failure. However, in most delay-time models the delay-time distribution is a property of the system itself, whereas in this paper the time from the occurrence of a system fault to the next triggering event depends on a factor external to and independent of the system. Besides, the system damage does not occur as a single event, but as two combined ones (a system fault and a triggering event). Nevertheless, the problem considered in the current paper can be defined in terms of a delay-time model, where the delay time elapses from an occurrence of a system fault to the next triggering event causing the system damage. As 
International Journal of Mathematical, Engineering and Management Sciences

Vol. 6, No. 1, 396-415, 2021

https://doi.org/10.33889/IJMEMS.2021.6.1.025

shown further in the paper, this delay time has exponential distribution with parameter $\mathrm{p} \lambda$. The issue of delay-time modeling is comprehensively treated in Werbinska-Wojciechowska (2019) - a freshly published monograph. For surveys of recent and older results concerning this topic, refer to Wang (2012), and Cha and Finkelstein (2019).

Problems similar to the one studied here have been investigated by several authors, e.g. Lienhardt et al. (2008), Huynh et al. (2011), and Badia et al. (2018). The first of these papers addresses the issue of choosing an appropriate maintenance policy to detect hidden failures of warning devices or backup components that do not interrupt normal aircraft operation. In the second one the optimal time between inspections is found numerically for a system with hidden failures caused by random shocks and gradual degradation modeled by a gamma process. The third paper investigates a system with two types of failures - minor (followed by repairs) and catastrophic (followed by replacements), where repairs are performed according to a General Polya process. The assumptions adopted in the above cited papers, although encompassing a wide spectrum of technical systems, do not allow for analytical solution of the optimal inspection policy problem. The possibility of analytical approach to this problem is the main advantage of the inspection/maintenance model proposed in this paper. To the best of its author's knowledge, the solution presented here cannot be found in the relevant literature, hence it is a new development in the area of maintenance modeling and optimization.

It should be noted that the proposed model must not be confused with age replacement models, where a system is replaced (or renewed) when it reaches age $\mathrm{T}$ or upon failure, whichever occurs first. For such models, preventive renewals make sense if the distribution of the system's time-tofailure (TTF) has the increasing failure rate (IFR). If TTF is exponentially distributed (constant failure rate), then, due to the lack-of-memory property, the only plausible maintenance policy is to replace the system upon its failure.

For better understanding, theoretical considerations will be illustrated by an example of a generic explosion prevention system (EPS) which, in order to perform its protective function reliably, has to undergo periodic inspection and maintenance. An explosion can be caused by the presence of combustible gas, dust or vapor, and triggered by an electric spark. Main functions of an EPS are suppressing an explosion and providing the proper venting. Guidelines for installing and operating EPS-s are given in the standard NFPA 69 (2019), published by National Fire Protection Association.

\section{Detailed Assumptions and Notation}

Main assumptions:

(i) A fault occurs after time $\mathrm{S}$ from putting the system into operation.

(ii) $\mathrm{S}$ is exponentially distributed with parameter $\mu$.

(iii) Damage can only happen to a faulty system and is caused by a triggering event.

(iv) A triggering event causes damage with probability $\mathrm{p}$.

(v) Triggering events occur according to a Poisson process with rate $\lambda$.

(vi) Occurrences of system faults are independent of triggering events.

(vii) System faults are not self-revealing, contrary to damages which manifest themselves immediately. 
International Journal of Mathematical, Engineering and Management Sciences

Vol. 6, No. 1, 396-415, 2021

https://doi.org/10.33889/IJMEMS.2021.6.1.025

(viii) In order to decrease the possibility of system damage, periodic inspections are carried out for the purpose of detecting and removing system faults.

(ix) An inspection is planned after time $\mathrm{T}$ from each moment when the system is put into operation.

(x) If a system fault is detected during an inspection, the fault is removed and the system is again put into operation.

(xi) A damaged system is replaced by a new one.

It is assumed that the time unit for $\lambda, \mu$ and $\mathrm{T}$ is one month, i.e. $\lambda$ is the average number of triggering events per month and $1 / \mu$ is the expected value of $S$ in months.

Additional notations are

$\mathrm{T}$ - planned time to next inspection

$\mathrm{A}_{\mathrm{T}}$ - the event "system damage occurs up to time T", where time is measured from the moment when the system is last put into operation

$\mathrm{B}_{\mathrm{T}}$ - the event "system fault occurs up to time T", where time is measured as above

$\neg \mathrm{A}_{\mathrm{T}}, \neg \mathrm{B}_{\mathrm{T}}-$ negations of the events $\mathrm{A}_{\mathrm{T}}$ and $\mathrm{B}_{\mathrm{T}}$

$\tau-$ a random time that elapses from putting the system into operation to its damage, provided that the system is left unmaintained

$\mathrm{L}_{\mathrm{T}}$ - the length of one operation cycle, i.e. the time that elapses from putting the system into operation to the next planned inspection or system damage, whichever occurs first

$\mathrm{C}_{\mathrm{I}}$ - the cost of inspection

$\mathrm{C}_{\mathrm{R}}$ - the cost of repair of a faulty system

$\mathrm{C}_{\mathrm{D}}$ - the cost of system damage and the ensuing replacement

$c_{a}(T)$ - the long-run operating cost per unit time

As shown further, the considered maintenance model yields four optimal maintenance policies related to four different configurations of the parameters $\mathrm{p}, \lambda, \mu, \mathrm{C}_{\mathrm{I}}, \mathrm{C}_{\mathrm{R}}$ and $\mathrm{C}_{\mathrm{D}}$. This is due to the fact that the cost functional $c_{a}(T)$ is different in the cases $p \lambda=\mu$ and $p \lambda \neq \mu$, as In consequence, the above parameters' values assumed for the example system will vary depending on the currently analyzed configuration.

\section{General Auxiliary Formulas}

$\operatorname{Pr}\left(\neg \mathrm{A}_{\mathrm{T}}\right), \mathrm{E}\left(\mathrm{L}_{\mathrm{T}}\right)$ and $\mathrm{c}_{\mathrm{a}}(\mathrm{T})$ defined in the previous section will be given by formulas (6), (17) and (18) respectively. Their derivations are presented below, starting with $\operatorname{Pr}\left(\neg \mathrm{A}_{\mathrm{T}}\right)$. Since $\neg \mathrm{B}_{\mathrm{T}} \subset \neg \mathrm{A}_{\mathrm{T}}$, we have:

$$
\begin{aligned}
& \operatorname{Pr}\left(\neg A_{T}\right)=\operatorname{Pr}\left[\left(\neg A_{T}\right) \cap B_{T}\right]+\operatorname{Pr}\left[\left(\neg A_{T}\right) \cap\left(\neg B_{T}\right)\right] \\
& \quad=\int_{0}^{T} \operatorname{Pr}\left(\neg A_{T} \mid \begin{array}{l}
\text { fault occurs at time } s \\
\text { from start of cycle }
\end{array}\right) d F_{S}(s)+\operatorname{Pr}\left(\neg B_{T}\right)
\end{aligned}
$$

System faults and triggering events are independent, hence for $\mathrm{s} \leq \mathrm{T}$ we have: 
International Journal of Mathematical, Engineering and Management Sciences

Vol. 6, No. 1, 396-415, 2021

https://doi.org/10.33889/IJMEMS.2021.6.1.025

$$
\begin{aligned}
& \operatorname{Pr}\left(\neg A_{T} \mid \begin{array}{l}
\text { fault occurs at time } s \\
\text { from start of cycle }
\end{array}\right) \\
& =\sum_{k=0}^{\infty} \operatorname{Pr}\left(\neg A_{T} \mid \begin{array}{c}
\text { fault occurs at time } s, \\
\text { and } k \text { triggering events } \\
\text { occur from s to } T
\end{array}\right) \operatorname{Pr}\left(\begin{array}{c}
k \text { triggering events } \\
\text { occur from s to } T
\end{array}\right) \\
& =\sum_{k=0}^{\infty}(1-p)^{k} \frac{\lambda^{k}(T-s)^{k}}{k !} \exp [-\lambda(T-s)]=\exp [-\lambda(T-s)] \sum_{k=0}^{\infty} \frac{[(1-p) \lambda(T-s)]^{k}}{k !} \\
& =\exp [-\lambda(T-s)] \exp [(1-p) \lambda(T-s)]=\exp [-p \lambda(T-s)]
\end{aligned}
$$

Since $\operatorname{Pr}\left(\neg \mathrm{B}_{\mathrm{T}}\right)=1-\mathrm{F}_{\mathrm{S}}(\mathrm{T})$, formula (1) converts to

$$
\operatorname{Pr}\left(\neg A_{T}\right)=\int_{0}^{T} \exp [-p \lambda(T-s)] d F_{S}(s)+1-F_{S}(T)
$$

The main assumption 2 states that

$$
F_{S}(s)=1-\exp (-\mu s)
$$

hence $\left.\operatorname{Pr}\left[\left(\neg \mathrm{A}_{\mathrm{T}}\right) \cap \mathrm{B}_{\mathrm{T}}\right)\right]$, equal to the integral in (3), is computed as follows:

$$
\begin{aligned}
& \operatorname{Pr}\left[\left(\neg A_{T}\right) \cap B_{T}\right]=\int_{0}^{T} \exp [-p \lambda(T-s)] d F_{S}(s) \\
& =\int_{0}^{T} \exp [-p \lambda(T-s)] \mu \exp (-\mu s) d s=\mu \int_{0}^{T} \exp (-p \lambda T+p \lambda s-\mu s) d s \\
& =\mu \exp (-p \lambda T) \int_{0}^{T} \exp [(p \lambda-\mu) s] d s \\
& =\left\{\begin{array}{c}
\mu \exp (-\mu T) T, p \lambda=\mu \\
\mu \exp (-p \lambda T)\left[\frac{1}{p \lambda-\mu} \exp [(p \lambda-\mu) s]\right]_{s=0}^{s=T}, p \lambda \neq \mu
\end{array}\right. \\
& =\left\{\begin{array}{c}
\mu T \exp (-\mu T), p \lambda=\mu \\
\frac{\mu}{p \lambda-\mu} \exp (-p \lambda T)[\exp (p \lambda T) \exp (-\mu T)-1], p \lambda \neq \mu
\end{array}\right. \\
& =\left\{\begin{array}{c}
\mu T \exp (-\mu T), p \lambda=\mu \\
\frac{\mu}{p \lambda-\mu}[\exp (-\mu T)-\exp (-p \lambda T)], p \lambda \neq \mu
\end{array}\right.
\end{aligned}
$$

Therefore, (3) becomes the following equality: 
International Journal of Mathematical, Engineering and Management Sciences

Vol. 6, No. 1, 396-415, 2021

https://doi.org/10.33889/IJMEMS.2021.6.1.025

$$
\begin{aligned}
& \operatorname{Pr}\left(\neg A_{T}\right)=1-\exp (-\mu T)+\left\{\begin{array}{c}
\mu T \exp (-\mu T), p \lambda=\mu \\
\frac{\mu}{p \lambda-\mu}[\exp (-\mu T)-\exp (-p \lambda T)], p \lambda \neq \mu
\end{array}\right. \\
& =\left\{\begin{array}{c}
(1+\mu T) \exp (-\mu T), p \lambda=\mu \\
\frac{p \lambda}{p \lambda-\mu} \exp (-\mu T)-\frac{\mu}{p \lambda-\mu} \exp (-p \lambda T), p \lambda \neq \mu
\end{array}\right.
\end{aligned}
$$

Our next goal is to compute $\mathrm{E}\left(\mathrm{L}_{\mathrm{T}}\right)$. For this purpose, we will need a formula for the distribution function of $\tau$, i.e. $\operatorname{Pr}(\tau \leq \mathrm{t})$. We have:

$$
\begin{aligned}
& \operatorname{Pr}(\tau \leq t)=\operatorname{Pr}\left(\begin{array}{c}
\text { damage occurs up to time } t \\
\text { from start of operation }
\end{array}\right) \\
& =\sum_{k=1}^{\infty} \operatorname{Pr}\left(\begin{array}{c}
\text { damage is triggered } \\
\text { by the } k-\text { th event } \\
\text { occuring after a system fault } \\
\text { up to time t from start of operation, } \\
\text { but not by earlier event }
\end{array}\right)
\end{aligned}
$$

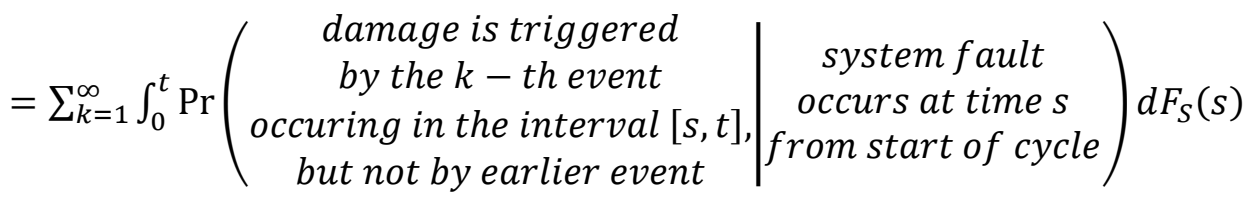

$$
\begin{aligned}
& =\sum_{k=1}^{\infty} \int_{0}^{t} p(1-p)^{k-1} \operatorname{Pr}\left(X_{1}+\cdots+X_{k} \leq t-s\right) d F_{S}(s)
\end{aligned}
$$

Where, $X_{1}$ is the time between $s$ and the first event occurring after $\mathrm{s}$, and $\mathrm{X}_{\mathrm{i}}$ - the time between the (i-1)-th and $i$-th event, $i \geq 2$. By main assumption 5, the sum $X_{1}+\ldots+X_{k}$ has Erlang distribution with parameters $\lambda$ and $\mathrm{k}$, i.e.

$$
\operatorname{Pr}\left(X_{1}+\cdots+X_{k} \leq t-s\right)=1-\sum_{j=0}^{k-1} \frac{1}{j !}(\lambda(t-s))^{j} \exp (-\lambda(t-s))
$$

From (7) and (8) we obtain:

$$
\operatorname{Pr}(\tau \leq t)=\int_{0}^{t} \sum_{k=1}^{\infty} p(1-p)^{k-1}\left[1-\sum_{j=0}^{k-1} \frac{1}{j !}(\lambda(t-s))^{j} \exp (-\lambda(t-s))\right] d F_{S}(s)
$$

Let us convert the expression under the integral in (9) to a simpler form. 


$$
\begin{aligned}
& \sum_{k=1}^{\infty} p(1-p)^{k-1}\left[1-\sum_{j=0}^{k-1} \frac{1}{j !}(\lambda(t-s))^{j} \exp (-\lambda(t-s))\right] \\
& =\sum_{k=1}^{\infty} p(1-p)^{k-1}-\exp (-\lambda(t-s)) \sum_{k=1}^{\infty} p(1-p)^{k-1} \sum_{j=0}^{k-1} \frac{1}{j !}(\lambda(t-s))^{j} \\
& p+ \\
& =1-\exp (-\lambda(t-s)) \times\left[\begin{array}{c}
p(1-p)[1+\lambda(t-s)]+ \\
p(1-p)^{2}\left[1+\lambda(t-s)+\frac{1}{2 !} \lambda^{2}(t-s)^{2}\right]+ \\
p(1-p)^{3}\left[1+\cdots+\frac{1}{3 !} \lambda^{3}(t-s)^{3}\right]+\cdots
\end{array}\right] \\
& =1-\exp (-\lambda(t-s)) \sum_{j=1}^{\infty} \frac{1}{(j-1) !}(\lambda(t-s))^{j-1} \sum_{k=j}^{\infty} p(1-p)^{k-1} \\
& =1-\exp (-\lambda(t-s)) \sum_{j=1}^{\infty} \frac{1}{(j-1) !}(\lambda(1-p)(t-s))^{j-1} \\
& =1-\exp (-\lambda(t-s)) \exp (\lambda(1-p)(t-s)) \\
& =1-\exp (-\lambda p(t-s))
\end{aligned}
$$

The second and the fourth of the above equalities follow from the formula for the sum of a geometric series. Substituting the sum under the integral in (9) according to (10) yields:

$$
\begin{aligned}
& \operatorname{Pr}(\tau \leq t)=\int_{0}^{t}[1-\exp (-\lambda p(t-s))] d F_{S}(s) \\
& \quad=F_{S}(t)-\int_{0}^{t} \exp (-\lambda p(t-s)) d F_{S}(s)
\end{aligned}
$$

It is convenient to compute $\mathrm{E}\left(\mathrm{L}_{\mathrm{T}}\right)$ using $\operatorname{Pr}(\tau>\mathrm{t})$ which, in view of $(11)$, is given by

$$
\operatorname{Pr}(\tau>t)=1-F_{S}(t)+\int_{0}^{t} \exp (-\lambda p(t-s)) d F_{S}(s)
$$

The definition of an operation cycle and formula (12) yield that

$$
\begin{aligned}
& E\left(L_{T}\right)=E[\min (\tau, T)]=\int_{0}^{T} \operatorname{Pr}(\tau>t) d t \\
& =\int_{0}^{T}\left[1-F_{S}(t)\right] d t+\int_{0}^{T} \int_{0}^{t} \exp (-\lambda p(t-s)) d F_{S}(s) d t
\end{aligned}
$$

We have: 
International Journal of Mathematical, Engineering and Management Sciences

Vol. 6, No. 1, 396-415, 2021

https://doi.org/10.33889/IJMEMS.2021.6.1.025

$$
\begin{aligned}
& \int_{0}^{T} \int_{0}^{t} \exp (-\lambda p(t-s)) d F_{S}(s) d t=\int_{0}^{T} \int_{S}^{T} \exp (-\lambda p(t-s)) d t d F_{S}(s) \\
& \quad=\int_{0}^{T} \exp (\lambda p s) \int_{S}^{T} \exp (-\lambda p t) d t d F_{S}(s) \\
& =\int_{0}^{T} \exp (\lambda p s) \frac{1}{\lambda p}[\exp (-\lambda p s)-\exp (-\lambda p T)] d F_{S}(s) \\
& =\frac{1}{\lambda p} \int_{0}^{T}[1-\exp (-\lambda p(T-s))] d F_{S}(s) .
\end{aligned}
$$

Hence

$$
E\left(L_{T}\right)=\int_{0}^{T}\left[1-F_{S}(t)\right] d t+\frac{1}{\lambda p} F_{S}(T)-\frac{1}{\lambda p} \int_{0}^{T} \exp (-\lambda p(T-s)) d F_{S}(s)
$$

Since $S$ is exponentially distributed, (14) converts to:

$$
\begin{gathered}
E\left(L_{T}\right)=\int_{0}^{T} \exp (-\mu t) d t+\frac{1}{\lambda p}[1-\exp (-\mu T)]+ \\
-\frac{\mu}{\lambda p} \int_{0}^{T} \exp (-\lambda p(T-s)) \exp (-\mu s) d s
\end{gathered}
$$

It holds that

$$
\begin{gathered}
\int_{0}^{T} \exp (-\lambda p(T-s)) \exp (-\mu s) d s=\exp (-p \lambda T) \int_{0}^{T} \exp ((p \lambda-\mu) s) d s \\
=\left\{\begin{array}{c}
\frac{1}{p \lambda-\mu}[\exp (-\mu T)-\exp (-p \lambda T)], p \lambda \neq \mu \\
T \exp (-\mu T), p \lambda=\mu
\end{array}\right.
\end{gathered}
$$

In consequence, we obtain

$$
E\left(L_{T}\right)=\left\{\begin{array}{c}
\left(\frac{1}{\mu}+\frac{1}{p \lambda}\right)[1-\exp (-\mu T)]+ \\
-\frac{\mu}{p \lambda(p \lambda-\mu)}[\exp (-\mu T)-\exp (-p \lambda T)], \quad p \lambda \neq \mu \\
\left(\frac{2}{\mu}\right)[1-\exp (-\mu T)]-T \exp (-\mu T), \quad p \lambda=\mu
\end{array}\right.
$$


International Journal of Mathematical, Engineering and Management Sciences

Vol. 6, No. 1, 396-415, 2021

https://doi.org/10.33889/IJMEMS.2021.6.1.025

$$
=\left\{\begin{array}{c}
\frac{1}{\mu}+\frac{1}{p \lambda}+\frac{\mu}{p \lambda(p \lambda-\mu)} \exp (-p \lambda T)+ \\
-\frac{p \lambda}{\mu(p \lambda-\mu)} \exp (-\mu T), p \lambda \neq \mu \\
\frac{2}{\mu}-\left(\frac{2}{\mu}+T\right) \exp (-\mu T), p \lambda=\mu
\end{array}\right.
$$

In the last part of this section we will construct a formula for the long-run operating cost per unit time, using $\operatorname{Pr}\left(\neg \mathrm{A}_{\mathrm{T}}\right)$ and $\mathrm{E}\left(\mathrm{L}_{\mathrm{T}}\right)$ given by (6) and (17). Clearly, the time points in which the system is put into operation (following an inspection or damage) constitute a renewal process. From the elementary renewal theorem (see Gertsbakh, 2000) it follows that

$c_{a}(T)=\frac{C_{I} \operatorname{Pr}\left(\neg A_{T}\right)+C_{R} \operatorname{Pr}\left(\left(\neg A_{T}\right) \cap B_{T}\right)+C_{D} \operatorname{Pr}\left(A_{T}\right)}{E\left(L_{T}\right)}$

Indeed, the above theorem implies that $c_{a}(T)$ is equal to the expected cost in one cycle divided by its expected length. To obtain the numerator in (18) let us note that 1) an inspection is performed at time $\mathrm{T}$ provided that damage does not occur up to $\mathrm{T} ; 2$ ) if, prior to an inspection, the system becomes faulty, it undergoes a repair; 3 ) if a damage occurs up to time $T$, the $\operatorname{cost} C_{D}$ is incurred.

In the next two sections we will formulate conditions to be fulfilled so that $c_{a}(T)<c_{a}(\infty)$ for certain $\mathrm{T}>0$, i.e. the inspection policy with period $\mathrm{T}$ is better than the "wait until damage and then replace" policy. We will then derive equations for the optimal $\mathrm{T}$ minimizing $\mathrm{c}_{\mathrm{a}}(\mathrm{T})$. Due to different expressions for $\operatorname{Pr}\left(\neg \mathrm{A}_{\mathrm{T}}\right)$ and $\mathrm{E}\left(\mathrm{L}_{\mathrm{T}}\right)$, the cases $\mathrm{p} \lambda=\mu$ and $\mathrm{p} \lambda \neq \mu$ have to be considered separately.

\section{Analysis of the Case $p \lambda=\mu$}

From (5), (6), (17), and (18) we have:

$C_{a}(T)=\frac{C_{D}-\left(C_{D}-C_{I}\right)(1+\mu T) \exp (-\mu T)+C_{R} \mu T \exp (-\mu T)}{\frac{2}{\mu}-\left(\frac{2}{\mu}+T\right) \exp (-\mu T)}=\frac{C_{D} \exp (\mu T)-\left(C_{D}-C_{I}\right)(1+\mu T)+C_{R} \mu T}{\frac{2}{\mu} \exp (\mu T)-\left(\frac{2}{\mu}+T\right)}$

Differentiating $\mathrm{c}_{\mathrm{a}}(\mathrm{T})$ we obtain:

$$
\begin{aligned}
& \frac{d c_{a}(T)}{d T}=\frac{\left[\mu C_{D} \exp (\mu T)-\mu\left(C_{D}-C_{R}-C_{I}\right)\right]\left[\frac{2}{\mu} \exp (\mu T)-\left(\frac{2}{\mu}+T\right)\right]}{\left[\frac{2}{\mu} \exp (\mu T)-\left(\frac{2}{\mu}+T\right)\right]^{2}}+ \\
& -\frac{\left[C_{D} \exp (\mu T)-\left(C_{D}-C_{I}\right)(1+\mu T)+C_{R} \mu T\right][2 \exp (\mu T)-1]}{\left[\frac{2}{\mu} \exp (\mu T)-\left(\frac{2}{\mu}+T\right)\right]^{2}} \\
& =\frac{\left(C_{D}-C_{I}\right)[1+2 \mu T \exp (\mu T)]-C_{D}(1+\mu T) \exp (\mu T)+2 C_{R}[\exp (\mu T)-1-\mu T \exp (\mu T)]}{\left[\frac{2}{\mu} \exp (\mu T)-\left(\frac{2}{\mu}+T\right)\right]^{2}} \\
& =\frac{\left(C_{D}-C_{I}\right)[\exp (-\mu T)+2 \mu T]-C_{D}(1+\mu T)+2 C_{R}[1-\mu T-\exp (-\mu T)]}{\left[\frac{2}{\mu} \exp (\mu T)-\left(\frac{2}{\mu}+T\right)\right]^{2} \exp (-\mu T)}
\end{aligned}
$$


International Journal of Mathematical, Engineering and Management Sciences

Vol. 6, No. 1, 396-415, 2021

https://doi.org/10.33889/IJMEMS.2021.6.1.025

\section{Lemma 1}

If $\mathrm{C}_{\mathrm{D}} \leq 2\left(\mathrm{C}_{\mathrm{I}}+\mathrm{C}_{\mathrm{R}}\right)$ then $\mathrm{c}_{\mathrm{a}}(\mathrm{T})$ decreases in $\mathrm{T}$ for $\mathrm{T}>0$.

Proof: Let $\mathrm{f}(\mathrm{T})$ be the numerator of $\mathrm{dc}_{\mathrm{a}}(\mathrm{T}) / \mathrm{dT}$ given by (20), i.e.

$f(T)=\left(C_{D}-C_{I}\right)[\exp (-\mu T)+2 \mu T]-C_{D}(1+\mu T)+2 C_{R}[1-\mu T-\exp (-\mu T)]$

If $C_{D} \leq 2\left(C_{I}+C_{R}\right)$ then

$$
\begin{aligned}
& f(T)=\left(C_{D}-2 C_{I}-2 C_{R}\right) \exp (-\mu T)+ \\
& \quad+\left(C_{D}-2 C_{I}-2 C_{R}\right) \mu T+2 C_{R}-C_{D}+C_{I} \exp (-\mu T)< \\
& \quad<\left(C_{D}-2 C_{I}-2 C_{R}\right)[\exp (-\mu T)+\mu T]+2 C_{R}-C_{D}+2 C_{I}= \\
& \quad=\left(C_{D}-2 C_{I}-2 C_{R}\right)[\exp (-\mu T)+\mu T-1] \leq 0
\end{aligned}
$$

The inequalities in (22) hold due to the fact that $\exp (-\mu \mathrm{T})<2$ and $\exp (-\mu \mathrm{T}) \geq 1-\mu \mathrm{T}$. Thus, in view of (20), the first derivative of $c_{a}(T)$ is strictly negative, which ends the proof.

Lemma 1 is illustrated in Figure 1, by the shape of $c_{a}(T)$, for the example system with the following parameters: $C_{I}=5, C_{R}=15, C_{D}=30, p \lambda=\mu=0.2$. Note that $c_{a}(T) \rightarrow \infty$ if $T \rightarrow 0$, as follows from (19).

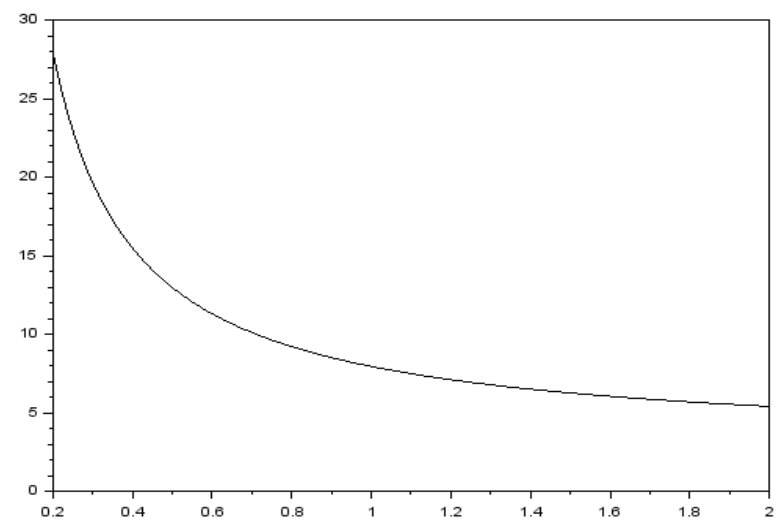

Figure 1. The shape of $c_{a}(T)$ as given by (19) if $C_{D} \leq 2\left(C_{I}+C_{R}\right)$.

Corollary: Under the assumption of Lemma 1 the optimal maintenance policy is "do not perform inspections or repairs and replace the system only after its damage".

\section{Lemma 2}

If $C_{D}>2\left(C_{I}+C_{R}\right)$ then $d_{a}(T) / d T$ is negative for $T \in\left[0, T^{*}\right)$, equal to 0 for $T=T^{*}$, and positive for $T>T^{*}$, where $T^{*}$ is the only solution of the equation $f(T)=0, f(T)$ being defined by $(21)$. 
International Journal of Mathematical, Engineering and Management Sciences

Vol. 6, No. 1, 396-415, 2021

https://doi.org/10.33889/IJMEMS.2021.6.1.025

Proof: The function $\mathrm{f}(\mathrm{T})$ is strictly convex because its second derivative, equal to $\left(C_{D}-C_{I}-2 C_{R}\right) \mu^{2} \exp (-\mu T)$, is positive. Thus, if there exists $T^{\prime}$ such that $f^{\prime}\left(T^{\prime}\right)=0$, then $f(T)$ decreases for $T<T^{\prime}$, attains its only minimum at $T^{\prime}$, and increases for $T>T^{\prime}$. We have

$$
\begin{aligned}
\frac{d f(T)}{d T} & =\left(C_{D}-C_{I}\right)[2 \mu-\mu \exp (-\mu T)]-C_{D} \mu+2 C_{R}[\mu \exp (-\mu T)-\mu] \\
& =\left(C_{D}-2 C_{1}-2 C_{R}\right) \mu-\left(C_{D}-C_{I}-2 C_{R}\right) \mu \exp (-\mu T)
\end{aligned}
$$

which yields

$T^{\prime}=-\frac{1}{\mu} \ln \left(\frac{C_{D}-2 C_{I}-2 C_{R}}{C_{D}-C_{I}-2 C_{R}}\right)$

Since $C_{D}>2\left(C_{I}+C_{R}\right)$, the expression under the logarithm falls within the interval $(0,1)$, hence $T^{\prime}>0$. Because $f(0)=-C_{I}$ and $f(T)$ decreases for $T<T^{\prime}$, it holds that $f(T)<-C_{I}<0$ for $T \in\left[0, T^{\prime}\right]$. In turn, as follows from the first equality in (22), $f(T)>\left(C_{D}-2 C_{I}-2 C_{R}\right) \mu T+2 C_{R}-C_{D}$, hence $f\left(T^{\prime \prime}\right)>0$ for $T^{\prime}=\left(C_{D}-2 C_{R}\right) /\left(C_{D}-2 C_{I}-2 C_{R}\right) \mu$. Because $f(T)$ increases for $T>T$ ', there exists strictly one $T^{*} \in\left(T^{\prime}, T^{\prime \prime}\right)$ such that $f\left(T^{*}\right)=0$. Clearly, $f(T)<0$ for $T \in\left[0, T^{*}\right)$ and $f(T)>0$ for $T>T^{*}$. Thus, the lemma is proved.

Lemma 2 is illustrated in Figure 2, by the shape of $\mathrm{f}(\mathrm{T})$, for the example system with the following parameters: $\mathrm{C}_{\mathrm{I}}=5, \mathrm{C}_{\mathrm{R}}=15, \mathrm{C}_{\mathrm{D}}=150, \mathrm{p} \lambda=\mu=0.2$.

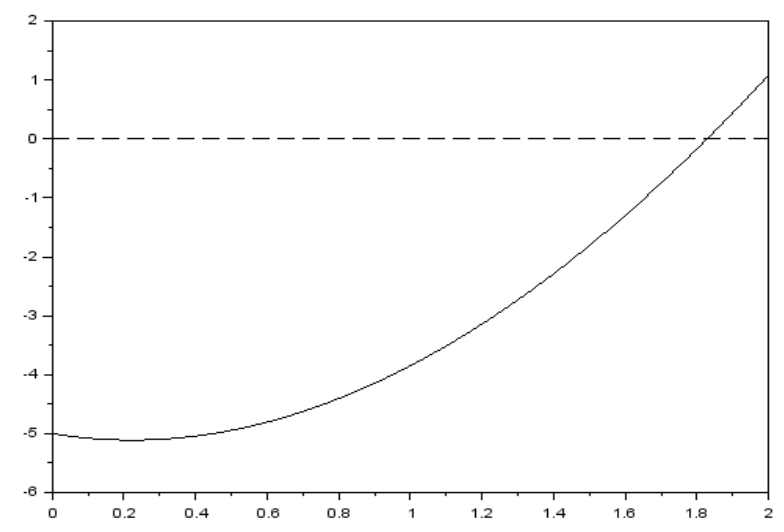

Figure 2. The shape of $f(T)$ defined by $(21)$ if $C_{D}>2\left(C_{I}+C_{R}\right)$.

Corollary: Under the assumption of Lemma 2, $\mathrm{c}_{\mathrm{a}}(\mathrm{T})$ decreases for $0<\mathrm{T}<\mathrm{T}^{*}$, attains minimum at $\mathrm{T}^{*}$, and increases for $T>T^{*}$, where $T^{*}$ is the only solution of the equation $f(T)=0$ (see Figure 5).

\section{Analysis of the Case $p \boldsymbol{\lambda} \neq \boldsymbol{\mu}$}

Using again (5), (6), (17), and (18), this time for $\mathrm{p} \lambda \neq \mu$, we obtain:

$$
c_{a}(T)=f(T) / g(T)
$$


International Journal of Mathematical, Engineering and Management Sciences

Vol. 6, No. 1, 396-415, 2021

where,

$$
\begin{aligned}
f(T) & =C_{D}-\left(C_{D}-C_{I}\right)\left[\frac{p \lambda}{p \lambda-\mu} \exp (-\mu T)-\frac{\mu}{p \lambda-\mu} \exp (-p \lambda T)\right] \\
& +C_{R} \frac{\mu}{p \lambda-\mu}[\exp (-\mu T)-\exp (-p \lambda T)] \\
g(T) & =\frac{1}{\mu}+\frac{1}{p \lambda}+\frac{\mu}{p \lambda(p \lambda-\mu)} \exp (-p \lambda T)-\frac{p \lambda}{\mu(p \lambda-\mu)} \exp (-\mu T)
\end{aligned}
$$

Let $f_{1}(T)=(p \lambda-\mu) f(T)$ and $g_{1}(T)=(p \lambda-\mu) g(T)$. We have:

$$
\begin{aligned}
c_{a}(T) & =\frac{f_{1}(T)}{g_{1}(T)}=\frac{C_{D}(p \lambda-\mu)-\left(C_{D}-C_{I}\right)[p \lambda \exp (-\mu T)-\mu \exp (-p \lambda T)]}{\frac{p \lambda}{\mu}[1-\exp (-\mu T)]-\frac{\mu}{p \lambda}[1-\exp (-p \lambda T)]} \\
& +\frac{C_{R} \mu[\exp (-\mu T)-\exp (-p \lambda T)]}{\frac{p \lambda}{\mu}[1-\exp (-\mu T)]-\frac{\mu}{p \lambda}[1-\exp (-p \lambda T)]}
\end{aligned}
$$

As in the previous section, we will differentiate $c_{a}(T)$, and the numerator of the resulting quotient will be the object of further analysis. Let $h_{1}(T)=f_{1}{ }^{\prime}(T) g_{1}(T)-f_{1}(T) g_{1}{ }^{\prime}(T)$, where $f_{1}$ ' and $g_{1}$ ' denote first derivatives of $\mathrm{f}_{1}$ and $\mathrm{g}_{1}$. It thus holds that

$d c_{a}(T) / d T=h_{1}(T) / g_{1}^{2}(T)$

We will now compute $h_{1}(T)$, which requires some effort.

$$
\begin{aligned}
& h_{1}(T)=\left[\begin{array}{c}
\left.\left(C_{D}-C_{I}\right) p \lambda \mu[\exp (-\mu T)-\exp (-p \lambda T)]+\right] \\
+C_{R} \mu[p \lambda \exp (-p \lambda T)-\mu \exp (-\mu T)]
\end{array}\right] \\
& \times\left(\frac{(p \lambda)^{2}}{p \lambda \mu}[1-\exp (-\mu T)]-\frac{\mu^{2}}{p \lambda \mu}[1-\exp (-p \lambda T)]\right) \\
& -\left[\begin{array}{c}
\left.C_{D}(p \lambda-\mu)-\left(C_{D}-C_{I}\right)[p \lambda \exp (-\mu T)-\mu \exp (-p \lambda T)]+\right] \\
+C_{R} \mu[\exp (-\mu T)-\exp (-p \lambda T)]
\end{array}\right] \\
& \quad \times[p \lambda \exp (-\mu T)-\mu \exp (-p \lambda T)]= \\
& =\left(C_{D}-C_{I}\right)\left[\begin{array}{c}
\left.(p \lambda)^{2} \exp (-\mu T)-(p \lambda)^{2} \exp (-2 \mu T)+\right] \\
-\mu^{2} \exp (-\mu T)+\mu^{2} \exp (-(p \lambda+\mu) T)
\end{array}\right] \\
& -\left(C_{D}-C_{I}\right)\left[\begin{array}{c}
(p \lambda)^{2} \exp (-p \lambda T)-(p \lambda)^{2} \exp (-(p \lambda+\mu) T) \\
-\mu^{2} \exp (-p \lambda T)+\mu^{2} \exp (-2 p \lambda T)
\end{array}\right] \\
& +\left(C_{D}-C_{I}\right)\left[(p \lambda)^{2} \exp (-2 \mu T)-2 p \lambda \mu \exp (-(p \lambda+\mu) T)+\mu^{2} \exp (-2 p \lambda T)\right] \\
& -C_{D}(p \lambda-\mu)[p \lambda \exp (-\mu T)-\mu \exp (-p \lambda T)] \\
& +C_{R} \mu[p \lambda \exp (-p \lambda T)-\mu \exp (-\mu T)] \times
\end{aligned}
$$


International Journal of Mathematical, Engineering and Management Sciences

Vol. 6, No. 1, 396-415, 2021

https://doi.org/10.33889/IJMEMS.2021.6.1.025

$$
\begin{aligned}
& \times\left(\frac{(p \lambda)^{2}}{p \lambda \mu}[1-\exp (-\mu T)]-\frac{\mu^{2}}{p \lambda \mu}[1-\exp (-p \lambda T)]\right) \\
- & C_{R} \mu[\exp (-\mu T)-\exp (-p \lambda T)][p \lambda \exp (-\mu T)-\mu \exp (-p \lambda T)]
\end{aligned}
$$

After appropriate reductions we obtain:

$$
\begin{aligned}
& h_{1}(T)=\left(C_{D}-C_{I}\right)(p \lambda-\mu) \times \\
& \quad \times[(p \lambda-\mu) \exp (-(p \lambda+\mu) T)+(p \lambda+\mu)(\exp (-\mu T)-\exp (-p \lambda T))] \\
& \quad-C_{D}(p \lambda-\mu)[p \lambda \exp (-\mu T)-\mu \exp (-p \lambda T)] \\
& +C_{R}\left[\mu^{2}+p \lambda \mu-\frac{\mu^{3}}{p \lambda}-(p \lambda)^{2}\right] \exp [-(p \lambda+\mu) T] \\
& +C_{R}\left[(p \lambda)^{2}-\mu^{2}\right] \exp (-p \lambda T)+C_{R} \mu\left(\frac{\mu^{2}}{p \lambda}-p \lambda\right) \exp (-\mu T)
\end{aligned}
$$

Let $\mathrm{h}(\mathrm{T})=\mathrm{h}_{1}(\mathrm{~T}) \exp [(\mathrm{p} \lambda+\mu) \mathrm{T}]$. We have:

$$
\begin{aligned}
& h(T)=\left(C_{D}-C_{I}\right)(p \lambda-\mu)[(p \lambda-\mu)+(p \lambda+\mu)(\exp (p \lambda T)-\exp (\mu T))] \\
& \quad-C_{D}(p \lambda-\mu)[p \lambda \exp (p \lambda T)-\mu \exp (\mu T)]+C_{R}\left[\mu^{2}+p \lambda \mu-\frac{\mu^{3}}{p \lambda}-(p \lambda)^{2}\right] \\
& \quad+C_{R} \mu\left(\frac{\mu^{2}}{p \lambda}-p \lambda\right) \exp (p \lambda T)+C_{R}\left[(p \lambda)^{2}-\mu^{2}\right] \exp (\mu T) \\
& =\left(C_{D}-C_{I}\right)(p \lambda-\mu)^{2}+C_{D}(p \lambda-\mu)[\mu \exp (p \lambda T)-p \lambda \exp (\mu T)] \\
& \quad-C_{I}(p \lambda-\mu)[p \lambda \exp (p \lambda T)-p \lambda \exp (\mu T)+\mu \exp (p \lambda T)-\mu \exp (\mu T)] \\
& \quad+C_{R} p \lambda \mu\left[1-\frac{\mu^{2}}{(p \lambda)^{2}}\right]+C_{R}\left[\mu^{2}-(p \lambda)^{2}\right] \\
& \quad+C_{R} p \lambda \mu\left(\frac{\mu^{2}}{(p \lambda)^{2}}-1\right) \exp (p \lambda T)+C_{R}\left[(p \lambda)^{2}-\mu^{2}\right] \exp (\mu T) \\
& =(p \lambda-\mu)^{2}\left(C_{D}-C_{I}\right)+C_{R}\left[\frac{\mu}{p \lambda}-1\right]\left[(p \lambda)^{2}-\mu^{2}\right] \\
& \quad+(p \lambda-\mu)\left(C_{D}-C_{I}\right)[\mu \exp (p \lambda T)-p \lambda \exp (\mu T)] \\
& \quad-(p \lambda-\mu) C_{I}[p \lambda \exp (p \lambda T)-\mu \exp (\mu T)] \\
& \quad-(p \lambda-\mu)(p \lambda+\mu) \frac{\mu}{p \lambda} C_{R} \exp (p \lambda T)+(p \lambda-\mu)(p \lambda+\mu) C_{R} \exp (\mu T) \\
& =(p \lambda-\mu)^{2}\left(C_{D}-C_{I}-C_{R} \frac{p \lambda+\mu}{p \lambda}\right) \\
& +(p \lambda-\mu)\left[\left(C_{D}-C_{I}-C_{R} \frac{p \lambda+\mu}{p \lambda}\right) \mu-C_{I} p \lambda\right] \exp (p \lambda T) \\
& -(p \lambda-\mu)\left[\left(C_{D}-C_{I}-C_{R} \frac{p \lambda+\mu}{p \lambda}\right) p \lambda-C_{I} \mu\right] \exp (\mu T) \\
& +(p)
\end{aligned}
$$


International Journal of Mathematical, Engineering and Management Sciences

Vol. 6, No. 1, 396-415, 2021

https://doi.org/10.33889/IJMEMS.2021.6.1.025

It will be now shown that $\mathrm{h}(\mathrm{T})$ fulfills the following two lemmas:

\section{Lemma 3}

The function $\mathrm{h}(\mathrm{T})$ is negative for $\mathrm{T} \geq 0$ if the following condition holds:

$C_{D} \leq C_{I} \max \left(\frac{p \lambda+\mu}{\mu}, \frac{p \lambda+\mu}{p \lambda}\right)+C_{R} \frac{p \lambda+\mu}{p \lambda}$

Proof: To begin with, let us compute $h(0)$. Putting $C_{X}=C_{D}-C_{I}-(p \lambda+\mu) C_{R} / p \lambda$ yields:

$$
\begin{aligned}
& h(0)=(p \lambda-\mu)^{2} C_{X}+(p \lambda-\mu)\left(\mu C_{X}-p \lambda C_{I}\right)-(p \lambda-\mu)\left(p \lambda C_{X}-\mu C_{I}\right) \\
& =(p \lambda-\mu)^{2} C_{X}-(p \lambda-\mu)^{2} C_{X}-(p \lambda-\mu)^{2} C_{I}=-(p \lambda-\mu)^{2} C_{I}<0
\end{aligned}
$$

Since $\mathrm{p} \lambda \neq \mu$, it is natural to consider the cases $\mathrm{p} \lambda>\mu$ and $\mathrm{p} \lambda<\mu$. Let us assume that $\mathrm{p} \lambda>\mu$ and $\mathrm{C}_{\mathrm{D}}$ fulfills an inequality stronger than (30), i.e.

$C_{D} \leq C_{R}(p \lambda+\mu) / p \lambda$

We will show that $\mathrm{dh}(\mathrm{T}) / \mathrm{dT}<0$ for $\mathrm{T}>0$. Indeed, from (29) we obtain:

$$
\begin{aligned}
& \frac{d h(T)}{d t}=(p \lambda-\mu) C_{X} p \lambda \mu[\exp (p \lambda T)-\exp (\mu T)]+ \\
& \quad+(p \lambda-\mu) C_{I}\left[(p \lambda)^{2} \exp (p \lambda T)-(\mu)^{2} \exp (\mu T)\right]<0
\end{aligned}
$$

The inequality in (33) holds because the expressions in parentheses and brackets are positive for $\mathrm{T}>0$, and $\mathrm{C}_{\mathrm{X}} \leq-\mathrm{C}_{\mathrm{I}}<0$ due to (32). From (33) it follows that $\mathrm{h}(\mathrm{T})$ is decreasing in $\mathrm{T}$ for $\mathrm{T}>0$, which, in view of (31), means that the lemma holds provided that (32) is fulfilled.

Let us now assume that $C_{D}$ fulfills (30) and the inequality opposite to (32). Since $p \lambda>\mu$, the maximum in (30) is equal to $(\mathrm{p} \lambda+\mu) / \mu$, hence we have:

$\frac{C_{R}(p \lambda+\mu)}{p \lambda}<C_{D} \leq \frac{C_{I}(p \lambda+\mu)}{\mu}+\frac{C_{R}(p \lambda+\mu)}{p \lambda}$

If we put $\mathrm{C}_{\mathrm{Y}}=\mathrm{C}_{\mathrm{D}}-\mathrm{C}_{\mathrm{R}}(\mathrm{p} \lambda+\mu) / \mathrm{p} \lambda$, (34) yields that $\mathrm{C}_{\mathrm{Y}}>0$ and

$\left(C_{Y}-C_{I}\right) \mu-C_{I} p \lambda \leq 0$

Since $\mathrm{p} \lambda>\mu$, the above inequality implies:

$\left[\left(C_{Y}-C_{I}\right) \mu-C_{I} p \lambda\right] \exp (p \lambda T) \leq\left[\left(C_{Y}-C_{I}\right) \mu-C_{I} p \lambda\right] \exp (\mu T)$

From (29) and (36), for T>0 we obtain:

$$
\begin{aligned}
& h(T) \leq(p \lambda-\mu)^{2}\left(C_{Y}-C_{I}\right)+(p \lambda-\mu)\left[\left(C_{Y}-C_{I}\right) \mu-C_{I} p \lambda\right] \exp (\mu T)+ \\
& \quad-(p \lambda-\mu)\left[\left(C_{Y}-C_{I}\right) p \lambda-C_{I} \mu\right] \exp (\mu T)= \\
& \quad=(p \lambda-\mu)^{2}\left(C_{Y}-C_{I}\right)-(p \lambda-\mu)^{2} C_{Y} \exp (\mu T) \leq-(p \lambda-\mu)^{2} C_{D}<0
\end{aligned}
$$


International Journal of Mathematical, Engineering and Management Sciences

Vol. 6, No. 1, 396-415, 2021

https://doi.org/10.33889/IJMEMS.2021.6.1.025

Thus, the lemma is true if (34) holds. This ends the proof for $\mathrm{p} \lambda>\mu$. The case $\mathrm{p} \lambda<\mu$ is handled analogously.

Corollary: Clearly, Lemma 3 also holds for $\mathrm{h}_{1}(\mathrm{~T})$, which means that, assuming (30), $\mathrm{dc}_{\mathrm{a}}(\mathrm{T}) / \mathrm{dT}$ is negative for $T>0$, i.e. $c_{a}(T)$ decreases in the interval $(0, \infty)$. Let us note that $c_{a}(T) \rightarrow \infty$ for $T \rightarrow 0$, as follows from (26). This means that under the assumption of Lemma 3 the optimal maintenance policy is "do not perform inspections or repairs and replace the system only after its damage".

The above corollary is illustrated in Figure 3, by the shape of $c_{a}(T)$, for the example system with the following parameters: $C_{I}=5, C_{R}=15, C_{D}=30, p \lambda=0.4, \mu=0.2$.

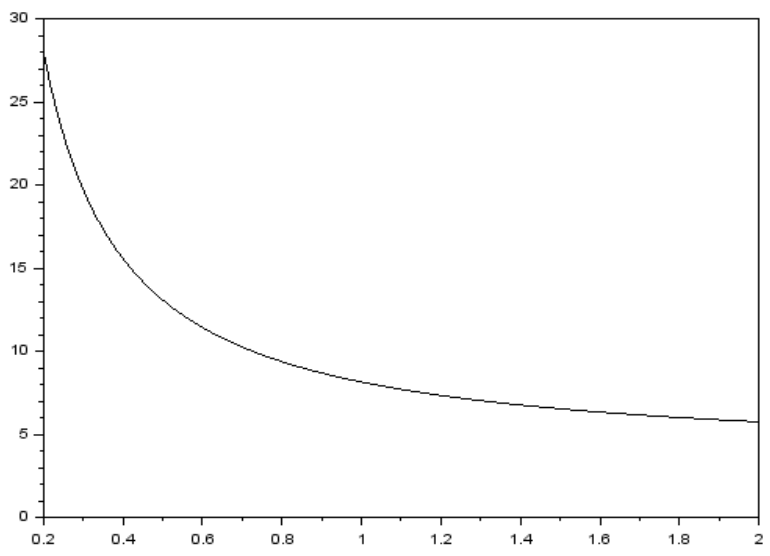

Figure 3. The shape of $c_{a}(T)$ as given by (26) under the condition (30).

\section{Lemma 4}

If the opposite of (30) holds, i.e.

$C_{D}>C_{I} \max \left(\frac{p \lambda+\mu}{\mu}, \frac{p \lambda+\mu}{p \lambda}\right)+C_{R} \frac{p \lambda+\mu}{p \lambda}$

then there exists strictly one $\mathrm{T}^{*}>0$ such that $\mathrm{h}(\mathrm{T})<0$ for $\mathrm{T} \in\left[0, \mathrm{~T}^{*}\right), \mathrm{h}\left(\mathrm{T}^{*}\right)=0$, and $\mathrm{h}(\mathrm{T})>0$ for $\mathrm{T}>\mathrm{T}^{*}$. Let us recall that $h(T)$ is defined by $(29)$.

Proof: As in Lemma 3, it is sufficient to prove Lemma 4 in the case $\mathrm{p} \lambda>\mu$. As already indicated, the maximum in $(38)$ is then equal to $(\mathrm{p} \lambda+\mu) / \mu$, and (38) converts to

$\left(C_{Y}-C_{I}\right) \mu-C_{I} p \lambda>0$

where, $C_{Y}=C_{D}-C_{R}(p \lambda+\mu) / p \lambda$. In consequence, $C_{Y}-C_{I}>C_{I} p \lambda / \mu>0$, and, since $p \lambda>\mu$, we have:

$\left(C_{Y}-C_{I}\right) p \lambda-C_{I} \mu>\left(C_{Y}-C_{I}\right) \mu-C_{I} p \lambda$

By (29), we have: 
International Journal of Mathematical, Engineering and Management Sciences

Vol. 6, No. 1, 396-415, 2021

https://doi.org/10.33889/IJMEMS.2021.6.1.025

$$
\begin{aligned}
& \frac{1}{(p \lambda-\mu)} \frac{d h(T)}{d T}=\left[\left(C_{Y}-C_{I}\right) p \lambda \mu-C_{I}(p \lambda)^{2}\right] \exp (p \lambda T)-\left[\left(C_{Y}-C_{I}\right) p \lambda \mu-C_{I}(\mu)^{2}\right] \exp (\mu T) \\
& =\exp (\mu T)\left[p \lambda\left(\left(C_{Y}-C_{I}\right) \mu-C_{I} p \lambda\right) \exp ((p \lambda-\mu) T)-\mu\left(\left(C_{Y}-C_{I}\right) p \lambda-C_{I} \mu\right)\right] \\
& =\exp (\mu T) p \lambda\left[\left(C_{Y}-C_{I}\right) \mu-C_{I} p \lambda\right]\left[\exp ((p \lambda-\mu) T)-\frac{\mu\left[\left(C_{Y}-C_{I}\right) p \lambda-C_{I} \mu\right]}{p \lambda\left[\left(C_{Y}-C_{I}\right) \mu-C_{I} p \lambda\right]}\right]
\end{aligned}
$$

Let $\alpha=\mu\left[\left(\mathrm{C}_{\mathrm{Y}}-\mathrm{C}_{\mathrm{I}}\right) \mathrm{p} \lambda-\mathrm{C}_{\mathrm{I}} \mu\right] / \mathrm{p} \lambda\left[\left(\mathrm{C}_{\mathrm{Y}}-\mathrm{C}_{\mathrm{I}}\right) \mu-\mathrm{C}_{\mathrm{I}} \mathrm{p} \lambda\right]$. In view of (39) and (40) it holds that $\alpha>0$. Let $\varphi(T)=\exp [(\mathrm{p} \lambda-\mu) \mathrm{T}]-\alpha$. If $\alpha \leq 1, \varphi(\mathrm{T})$ is positive for $\mathrm{T}>0$. In turn, if $\alpha>1, \varphi(\mathrm{T})$ is negative for $\mathrm{T} \in[0, \ln (\alpha) /(\mathrm{p} \lambda-\mu)$ ), equal to 0 for $\mathrm{T}=\ln (\alpha) /(\mathrm{p} \lambda-\mu)$, and positive for $\mathrm{T}>\ln (\alpha) /(\mathrm{p} \lambda-\mu)$. In addition, $\varphi(T)$ increases to infinity. Due to (39) and the assumption that $\mathrm{p} \lambda>\mu, \mathrm{dh}(\mathrm{T}) / \mathrm{dT}$ has the same properties as $\varphi(T)$. Thus, if $\alpha \leq 1$, then $\mathrm{h}(\mathrm{T})$ increases to infinity for $\mathrm{T}>0$. In turn, if $\alpha>1$, then $\mathrm{h}(\mathrm{T})$ decreases for $\mathrm{T} \in[0, \ln (\alpha) /(\mathrm{p} \lambda-\mu))$, and increases to infinity for $\mathrm{T}>\ln (\alpha) /(\mathrm{p} \lambda-\mu)$. Also, according to (31), $\mathrm{h}(0)=-(\mathrm{p} \lambda-\mu)^{2} \mathrm{C}_{\mathrm{I}}<0$.

From the above argument it follows that in either case $(\alpha \leq 1$ or $\alpha>1)$ there exists strictly one $T^{*}>0$ such that $\mathrm{h}(\mathrm{T})<0$ for $\mathrm{T} \in\left[0, \mathrm{~T}^{*}\right), \mathrm{h}\left(\mathrm{T}^{*}\right)=0$, and $\mathrm{h}(\mathrm{T})>0$ for $\mathrm{T}>\mathrm{T}^{*}$. The proof for the case $\mathrm{p} \lambda<\mu$ is analogous.

Lemma 4 is illustrated in Figure 4, by the shape of $h(T)$, for the example system with the following parameters: $C_{I}=5, C_{R}=15, C_{D}=150, p \lambda=0.4, \mu=0.2$. Let us note that $\alpha>1$, where $\alpha$ is defined in the proof of Lemma 4 , hence $h(T)$ first decreases to its minimum value, and then increases to infinity.

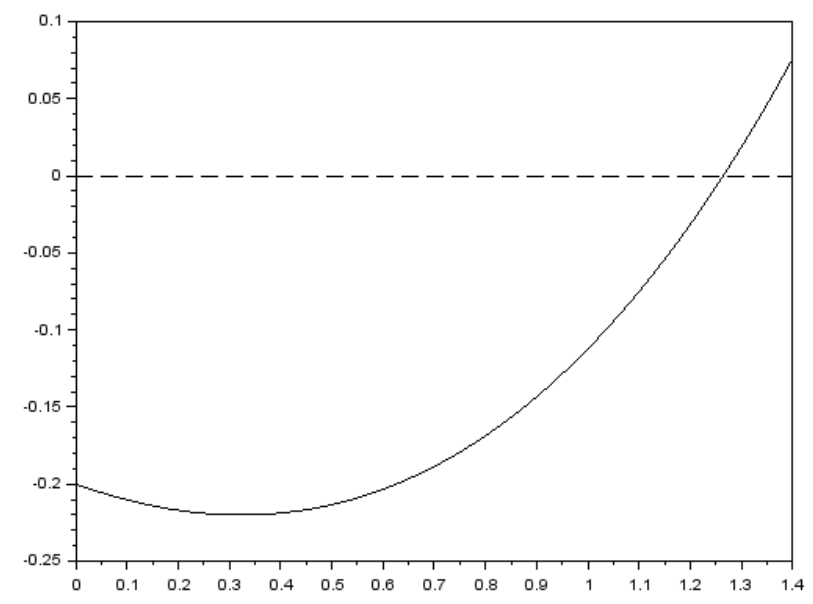

Figure 4. The shape of $h(T)$ defined by (29), for $\alpha>1$.

Corollary: Clearly, Lemma 4 also holds for $h_{1}(T)$ and, in consequence, for $\mathrm{dc}_{\mathrm{a}}(\mathrm{T}) / \mathrm{dt}$. This means that, under the assumption of Lemma $4, \mathrm{c}_{\mathrm{a}}(\mathrm{T})$ decreases for $0<\mathrm{T}<\mathrm{T}^{*}$, attains minimum at $\mathrm{T}^{*}$, and increases for $\mathrm{T}>\mathrm{T}^{*}$, where $\mathrm{T}^{*}$ is the only solution of the equation $\mathrm{h}(\mathrm{T})=0$ (see Figure 6 ). 
International Journal of Mathematical, Engineering and Management Sciences

Vol. 6, No. 1, 396-415, 2021

https://doi.org/10.33889/IJMEMS.2021.6.1.025

\section{Main Results}

The results of the two previous sections can be summarized as Theorems 1 and 2 which hold for the cases $\mathrm{p} \lambda=\mu$ and $\mathrm{p} \lambda \neq \mu$ respectively.

Theorem 1 (the case $\mathrm{p} \lambda=\mu$ ).

If $C_{D} \leq 2\left(C_{I}+C_{R}\right.$ then $c_{a}(T)$ decreases in $T$ for $T>0$, i.e. the optimal maintenance policy is "do not perform inspections or repairs and replace the system when damaged". In turn, if $C_{D}>2\left(C_{I}+C_{R}\right)$ then the optimal $T$ minimizing $c_{a}(T)$ is found by solving the equation $f(T)=0$, where $f(T)$ is given by $(21)$.

Theorem 2 (the case $\mathrm{p} \lambda \neq \mu$ ).

If

$C_{D} \leq C_{I} \max \left(\frac{p \lambda+\mu}{\mu}, \frac{p \lambda+\mu}{p \lambda}\right)+C_{R} \frac{p \lambda+\mu}{p \lambda}$

then $c_{a}(T)$ decreases in $T$ for $T>0$, i.e. optimal maintenance policy is "do not perform inspections or repairs and replace the system after its damage". In turn, if " $\leq$ " is replaced with " $>$ " in (42) then the optimal $T$ minimizing $c_{a}(T)$ is found by solving the equation $h(T)=0$, where $h(T)$ is given by (29).

The above theorems are illustrated in Figures 5 and 6 . For ease of comparison, the example system parameters are the same as for Figures 2 and 4 respectively. Let us note that $\mathrm{c}_{\mathrm{a}}(\mathrm{T})$ converges to a constant value, equal to $\mu \mathrm{C}_{\mathrm{D}} / 2$ if $\mathrm{p} \lambda=\mu$, or $\mathrm{p} \lambda \mu \mathrm{C}_{\mathrm{D}} /(\mathrm{p} \lambda+\mu)$ if $\mathrm{p} \lambda \neq \mu$. This can be easily calculated from (19) or (26).

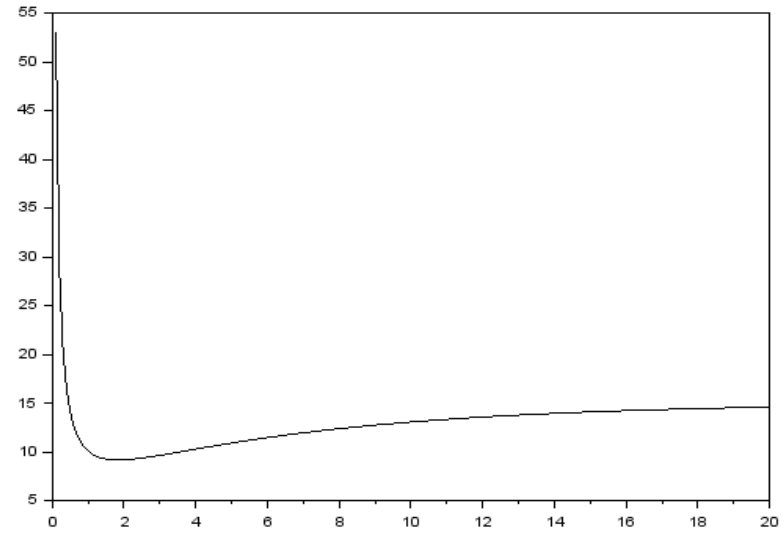

Figure 5. The shape of $c_{a}(T)$ for $p \lambda=\mu=0.2, C_{I}=5, C_{R}=15, C_{D}=150$. 
International Journal of Mathematical, Engineering and Management Sciences

Vol. 6, No. 1, 396-415, 2021

https://doi.org/10.33889/IJMEMS.2021.6.1.025

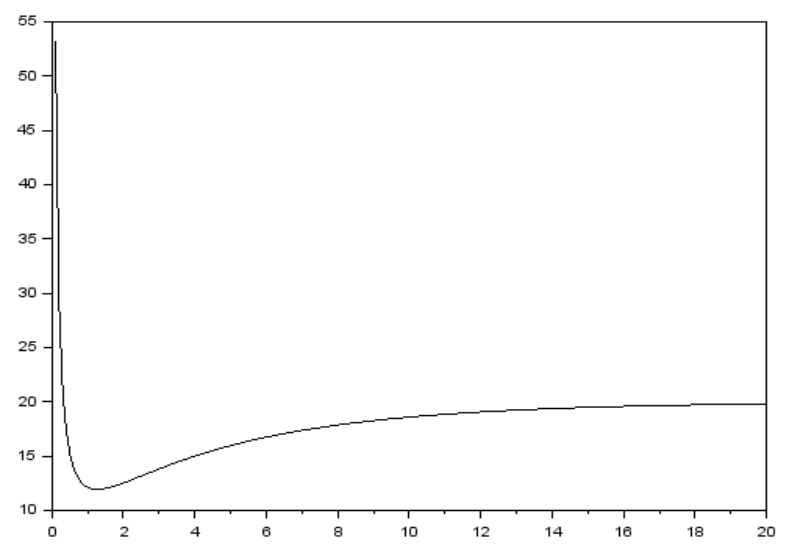

Figure 6. The shape of $c_{a}(T)$ for $p \lambda=0.4, \mu=0.2, C_{I}=5, C_{R}=15, C_{D}=150$.

\section{Conclusion}

The paper provides detailed reliability analysis of a technical system that can become faulty during operation, and is subject to repeatedly occurring hazardous events each of which can trigger damage to a faulty system. Assuming that time-to-fault is exponentially distributed and damage triggering events constitute a Poisson process, it was manageable to formulate conditions for the system parameters under which periodic maintenance policy is better (with regard to the long run operating cost per unit time) than the policy "wait until damage happens, then replace". The respective conditions, valid for the cases $\mathrm{p} \lambda=\mu$ and $\mathrm{p} \lambda \neq \mu$, are given by Lemmas 2 and 4 , and repeated in Theorems 1 and 2. Also, it was possible to analytically derive fairly simple equations for the optimal time between inspections, i.e. the equations $f(T)=0$ and $h(T)=0$, where $f(T)$ and $h(T)$ are given by (21) and (29) respectively. These equations can easily be solved by a numerical method finding the zero of a function, e.g. the Newton-Raphson method. It can be seen in the proofs of Lemmas 2 and 4 that both $\mathrm{f}(\mathrm{T})$ and $\mathrm{h}(\mathrm{T})$ fulfill the conditions allowing to apply the Newton-Raphson procedure.

The model analyzed in this paper can be applied to optimize the maintenance process of many realworld technical systems, in particular a wide range of safety systems. Apart from the explosion protection systems, mentioned in the introduction, the results of the paper can be implemented for power system protection devices (line protection relays or generator protection equipment) subject to hidden faults which, in some randomly occurring circumstances, may cause widespread power outages. This problem is addressed in Bae and Thorp (1999). Another example of the model's application is an electronic equipment with protection device whose failures are hidden and, if undetected, lead to the protected equipment damage. Such a system is considered in Jiang et al. (2015). Last but not least, as discussed in the introduction, the model can also be applied to systems with delayed failures.

The author believes that it is possible to add some complexity to the above considered maintenance model without losing its analytical tractability. E.g. we can assume non-homogeneity of the Poisson process of the damage-triggering events, as well as multilevel faults and/or multiple fault modes. Analysis of the extended model will be a subject of future work. 
International Journal of Mathematical, Engineering and Management Sciences

Vol. 6, No. 1, 396-415, 2021

https://doi.org/10.33889/IJMEMS.2021.6.1.025

\section{Conflict of Interest}

The author confirms that there is no conflict of interest pertaining to this publication.

\section{Acknowledgements}

This paper documents the research conducted by the author as an employee of the Systems Research Institute, Poland.

\section{References}

Abdel-Hameed, M. (1987). Inspection and maintenance policies of devices subject to deterioration. Advances in Applied Probability, 19(4), 917-931.

Abdel-Hameed, M. (1995). Correction to: Inspection and maintenance policies of devices subject to deterioration. Advances in Applied Probability, 27(2), 584.

Alaswad, S., \& Xiang, Y. (2017). A review on condition-based maintenance optimization models for stochastically deteriorating system. Reliability Engineering and System Safety, 157(1), 54-63.

Badía, F.G., Berradea, M.D., Cha, J.H., \& Lee, H. (2018). Optimal replacement policy under a general failure and repair model: minimal versus worse than old repair. Reliability Engineering and System Safety, 180, 362-372.

Bae, K., \& Thorp, J.S. (1999). A stochastic study of hidden failures in power system protection. Decision Support Systems, 24(3), 259-268.

Cao, W., Jia, X., Hu, Q., Zhao, J., \& Wu, Y. (2018). A literature review on selective maintenance for multiunit systems. Quality and Reliability Engineering International 34(5), 824-845.

Cha, J.H., \& Finkelstein, M. (2019). Stochastic modeling for systems with delayed failures. Reliability Engineering and System Safety, 188, 118-124.

Christer, A.H., \& Waller, W.M. (1984). Delay time models of industrial inspection maintenance problems. Journal of the Operational Research Society, 35(5), 401-406.

Duffuaa, S.O., \& Raouf, A. (2015). Planning and control of maintenance systems: modelling and analysis. Second Edition. Springer, Cham.

Gertsbakh, I. (2000). Reliability theory with applications to preventive maintenance. Springer, New York.

Jiang, X., Zhao, J., \& Li, Z. (2015). An optimal inspection policy for protection devices of electronic equipment using delay time model. International Journal of $u$ - and e-Service, Science and Technology. 8(8), 169-178.

Jardine, A.K., \& Tsang, A.H. (2006). Maintenance, replacement, and reliability: theory and applications. CRC Press, Taylor \& Francis Group, Boca Raton.

Guo, H., Szidarovszky, F., Gerokostopoulos, A., \& Niu, P. (2015). On determining optimal inspection interval for minimizing maintenance cost. In: 2015 Annual Reliability and Maintainability Symposium (RAMS), Palm Harbor, FL, USA, 1-7.

Huynh, K.T., Barros, A., Bérenguer, C., \& Castro, I. (2011). A periodic inspection and replacement policy for systems subject to competing failure modes due to degradation and traumatic events. Reliability Engineering and System Safety, 96 (4), 497-508.

Le, M.D., \& Tan, C.M. (2013). Optimal maintenance strategy of deteriorating system under imperfect maintenance and inspection using mixed inspection scheduling. Reliability Engineering and System Safety, 113, 21-29.

Lienhardt, B., Hugues, E., Bes, C., \& Noll, D. (2008). Failure-finding frequency for a repairable system subject to hidden failures. Journal of Aircraft, 45(5), 1804-1809. 
International Journal of Mathematical, Engineering and Management Sciences

Vol. 6, No. 1, 396-415, 2021

https://doi.org/10.33889/IJMEMS.2021.6.1.025

Mendes, A.A., Coit, D.W., \& Ribeiro, J.L.D. (2017). Optimal time interval between periodic inspections for a two-component cold standby multistate system. IEEE Transactions on Reliability, 66(2), 559-574.

Nakagawa, T., Mizutani, S., \& Chen, M. (2010). A summary of periodic and random inspection policies. Reliability Engineering and System Safety, 95(8), 906-911.

Nicolai, R.P., \& Dekker, R. (2008). Optimal maintenance of multi-component systems: a review. In: Complex System Maintenance Handbook. Springer Series in Reliability Engineering. Springer, London.

Pascual, R., Louit, D., \& Jardine, A.K. (2011). Optimal inspection intervals for safety systems with partial inspections. Journal of the Operational Research Society, 62(12), 2051-2062.

Peng, R., Liu, B., Zhai, Q., \& Wang, W. (2019). Optimal maintenance strategy for systems with two failure modes. Reliability Engineering and System Safety, 188, 624-632.

Sun, Q., Ye, Z., \& Chen, N. (2018). Optimal inspection and replacement policies for multi-unit systems subject to degradation. IEEE Transactions on Reliability, 67(1), 401-413.

Vasili, M., Sai, H.T., Sai, H., Ismail, N., \& Vasili, M. (2011). Maintenance optimization models: a review and analysis. In: Proceedings of the 2011 International Conference on Industrial Engineering and Operations Management, Kuala Lumpur, Malaysia, 22 - 24 Jan. 2011, 1131-1138.

Wang, W. (2012). An overview of the recent advances in delay-time-based maintenance modeling. Reliability Engineering and System Safety, 106, 165-178.

Wang, W. (2013). Models of inspection, routine service, and replacement for a serviceable one-component system. Reliability Engineering and System Safety, 116, 57-63.

Werbinska-Wojciechowska, S. (2019). Technical system maintenance. Delay-Time-Based Modelling, Springer Series in Reliability Engineering. Springer, Cham.

Zequiera, R.I., \& Berenguer, C. (2005). Optimal inspection policies with predictive and preventive maintenance. Engineering Optimization, 37(5), 541-550.

Zhao, X., Qian, C., \& Nakagawa, T. (2017). Comparisons of replacement policies with periodic times and repair numbers. Reliability Engineering and System Safety, 168, 161-170.

NFPA 69 - National Fire Protection Association (2019). Standard on explosion prevention systems. Available at https://www.nfpa.org/codes-and-standards/all-codes-and-standards/list-of-codes-andstandards/detail?code $=69$ 